\title{
Device from Same Lot Evaluated
} Evaluation Method

National Cancer Institute

\section{Source}

National Cancer Institute. Device from Same Lot Evaluated Evaluation Method. NCI

Thesaurus. Code C91933.

The investigation employed relevant empirical testing of the device of the same lot or batch than that of the suspected device in the reported adverse event in order to support the identification of possible causes for the adverse event. Testing was performed using the device retained by the manufacturer (i.e. was not shipped). Relevant testing would typically be based on test methods used for evaluating safety and performance as described in the latest relevant standards. 